\title{
Adesão ao tratamento anti-hipertensivo: dificuldades relatadas por indivíduos hipertensos*
}

\author{
Adherence to antibypertensive treatment: difficulties reported by bypertensive patients \\ Adhesión al tratamiento de hipertensión: dificultades relatadas por individuos hipertensos
}

\author{
Natalia Negreiros Figueiredo ${ }^{1}$, Leiko Asakura²
}

\begin{abstract}
RESUMO
Objetivos: Caracterizar pacientes hipertensos e conhecer suas principais dificuldades para aderir ao tratamento proposto pela equipe de saúde. Métodos: Os dados foram coletados em prontuários e por meio de entrevistas com 54 pacientes hipertensos. Resultados: Dentre os 54 pacientes hipertensos 66,7\% eram do sexo feminino, adultos com idade entre 30 e 90 anos. As médias da pressão arterial (mmHg) sistólica e diastólica foram 138 e 83, respectivamente, mas apenas metade dos pacientes apresentava pressão arterial controlada. Observou-se associação entre o conhecimento sobre a doença e o seguimento das orientações para o tratamento da hipertensão. A principal dificuldade relatada foi seguir dieta hipossódica. Comparando-se as orientações prescritas com aquelas que os pacientes referiram ter recebido, houve concordância em 37 casos. Conclusão: A comunicação entre os membros da equipe de saúde e os pacientes deve ser melhorada, e as pessoas envolvidas no tratamento da hipertensão precisam discutir as dificuldades encontradas e buscar formas de resolvê-las.
\end{abstract}

Descritores: Hipertensão; Cooperação do paciente; Terapêutica; Estilo de vida; Educação em saúde

\begin{abstract}
Objectives: To characterize hypertensive patients and to recognize the difficulties to comply with the proposed treatment by the health team. Methods: The data were collected from medical records and through interviews with 54 hypertensive patients. Results: Among the 54 hypertensive patients, $66.7 \%$ were adults female aged between 30 and 90 years. The mean blood pressures (mmHg), systolic and diastolic pressures, were 138 and 83, respectively, but only half of the patients had the blood pressure controlled. There was an association between the disease knowledge and the complying with guidelines for hypertension treatment. The main reported difficulty was following a low sodium diet. Comparing the guidelines prescribed to the patients with those actually followed there was agreement in 37 cases. Conclusion: The communication between members of the healthcare team and patients should be improved, and the people involved in the treatment of hypertension need to discuss the difficulties and find ways to resolve them.
\end{abstract}

Keywords: Hypertension; Patient compliance; Therapeutics; Life style; Health education

\section{RESUMEN}

Objetivos: Caracterizar pacientes hipertensos y conocer sus principales dificultades para adherir al tratamiento propuesto por el equipo de salud. Métodos: Los datos fueron recolectados en fichas y por medio de entrevistas con 54 pacientes hipertensos. Resultados: Entre los 54 pacientes hipertensos, 66,7\% eran del sexo femenino, adultos con edad entre 30 y 90 anos. Los promedios de la presión arterial (mmHg) sistólica y diastólica fueron 138 y 83, respectivamente, pero apenas la mitad de los pacientes presentaba presión arterial controlada. Se observó una asociación entre el conocimiento sobre la enfermedad y el seguimiento de las orientaciones para el tratamiento de la hipertensión. La principal dificultad relatada fue seguir una dieta con poca sal. Comparando las orientaciones prescritas con aquellas que los pacientes refirieron haber recibido, hubo concordancia en 37 casos. Conclusión: La comunicación entre los miembros del equipo de salud y los pacientes debe ser mejorada, y las personas envueltas en el tratamiento de la hipertensión necesitan discutir las dificultades encontradas y buscar formas de resolverlas.

Descriptores: Hipertensión; Cooperación del paciente; Terapéutica; Estilo de vida; Educación en salud

\footnotetext{
* Trabalho realizado em um Centro de Saúde (São Paulo, SP) como parte da monografia de conclusão de curso de Enfermagem apresentada ao Departamento de Enfermagem da Universidade Federal de São Paulo - UNIFESP - São Paulo (SP), Brasil.

1 Acadêmica de Enfermagem, Departamento de Enfermagem da Universidade Federal de São Paulo - UNIFESP - São Paulo (SP), Brasil.

${ }^{2}$ Professora Adjunto da Disciplina de Nutrição do Departamento de Medicina Preventiva da Universidade Federal de São Paulo - UNIFESP - São Paulo (SP), Brasil.
} 


\section{INTRODUÇÃO}

A hipertensão arterial (HA) é caracterizada pela presença de níveis de pressão arterial elevados, relacionados a alterações hormonais e, no metabolismo, a fenômenos tróficos; em estágio avançado causa lesões graves em órgãos-alvo como coração, rins, retina e cérebro que podem levar o indivíduo à dependência física ou até a falecer ${ }^{(1)}$.

A HA é ao mesmo tempo uma doença e um fator de risco independente para as doenças cardiovasculares principal causa de morte no mundo(2), como infarto agudo do miocárdio (IAM), insuficiência cardíaca congestiva e acidente vascular cerebral (AVC). Globalmente, a HA é responsável por $62 \%$ dos AVC e $49 \%$ dos $\operatorname{IAM}^{(3)}$.

Apesar do Brasil não dispor de estatísticas sobre a magnitude dessa doença em todo território nacional, estimativas realizadas baseados em estudos nas regiões Sul e Sudeste, de base populacional, indicam que, aproximadamente, $20 \%$ da população adulta brasileira têm $\mathrm{HA}^{(4)}$.

A doença, por não apresentar cura, exige tratamento adequado e para a vida inteira, a fim de se obter o controle da pressão arterial, a redução na incidência ou retardo na ocorrência de complicações cardiovasculares e a melhoria da qualidade de vida do portador.

O tratamento da hipertensão pode ser sem ou com medicamentos. O primeiro é, feito por meio do controle do peso, da melhora do padrão alimentar, da redução do consumo do sal, da moderação no consumo de bebidas alcoólicas, da prática regular de exercício físico, da abstenção do tabagismo e do controle do estresse psicoemocional; já o medicamentoso tem por base o uso de drogas prescritas pelo médico, conforme a gravidade do quadro ${ }^{(1)}$.

Em um estudo realizado em São Luís, Maranhão, dos 462 pacientes entrevistados, $75 \%$ aderiam ao tratamento medicamentoso; os pacientes que não seguiam o tratamento relataram como motivos principais: esquecimento, sensação de não necessidade do uso da medicação e efeitos colaterais provocados pela medicação ${ }^{(5)}$. A porcentagem de adesão foi menor ainda em um trabalho que investigou a prevalência de hipertensão em uma cidade de Minas Gerais $^{(6)}$ : 66,7\% dos indivíduos com conhecimento prévio da doença e medicados referiam fazer uso regular da medicação. Considerando que a adesão ao tratamento foi autorreferida em ambos trabalhos citados, este percentual pode estar superestimado. A adesão ao tratamento não medicamentoso parece ser mais difícil. Observando os relatos dos pacientes entrevistados verifica-se que o seguimento de uma dieta devidamente prescrita é negligenciada ${ }^{(7)}$.

Apesar da disponibilidade de tratamento efetivo, o controle da HA encontra-se distante do considerado ideal pela falta de adesão ao mesmo. Segundo a Organização Mundial da Saúde, a aderência a uma terapia de longo prazo é o grau em que o comportamento de uma pessoa, isto é, tomar um remédio, seguir uma dieta e mudar o estilo de vida, corresponde às recomendações do profissional que lhe prestou atendimento ${ }^{(8)}$.

É válido lembrar que a adesão ao tratamento sofre influência de fatores próprios do paciente, de sua relação com os membros da equipe multiprofissional de saúde e do contexto socioeconômico ${ }^{(9)}$.

Nesse sentido, os objetivos do presente estudo foram caracterizar os pacientes hipertensos e conhecer suas principais dificuldades para aderir ao tratamento proposto pela equipe de saúde.

\section{MÉTODOS}

Trata-se de um estudo descritivo de corte transversal, realizado de julho a setembro de 2008.

A amostra constituiu-se de pacientes aleatoriamente selecionados com base na pesquisa prévia aos prontuários disponibilizados daqueles que estavam em seguimento ambulatorial da Endocrinologia de um Centro de Saúde, localizado na cidade de São Paulo, nos quais constavam os critérios de inclusão, isto é, diagnóstico médico prévio de hipertensão arterial sistêmica, segundo os critérios da V Diretrizes Brasileiras de Hipertensão Arterial ${ }^{(1)}$ e idade igual ou maior que 18 anos.

Ainda, foram coletadas informações nos prontuários referentes ao tratamento proposto, como medicação, modificação no estilo de vida, tipo de orientação dietética e os valores das medidas de pressão arterial, peso e altura.

Em seguida, os pacientes foram entrevistados por um único pesquisador, antes da consulta médica, ainda na sala de espera. A entrevista seguiu um roteiro preestabelecido com perguntas abertas e fechadas sobre características sóciodemográficas, hábitos de vida e o tratamento para a hipertensão. Perguntou-se ainda o que os pacientes sabiam sobre a doença. Excluíram-se aqueles pacientes com prontuário incompleto em relação às variáveis de interesse, ou que estavam desacompanhados e apresentavam dificuldades para responder às perguntas, ou ainda, que se recusaram a participar do estudo.

Testou-se a associação entre as variáveis por meio do Qui-quadrado e utilizou-se o teste $\mathrm{t}$ de Student para a comparação dos valores médios das variáveis quantitativas; os valores de $\mathrm{p}<0,05$ foram considerados significantes.

A classificação do estado nutricional dos adultos (idade $<60$ anos) e dos idosos (idade $\geq 60$ anos), segundo o índice de massa corporal (IMC, $\mathrm{kg} / \mathrm{m}^{2}$ ), seguiu os critérios da Organização Mundial da Saúde ${ }^{(10)}$ e da Organização Panamericana de Saúde, citados por 
Marucci ${ }^{(11)}$, respectivamente.

Os pacientes foram informados dos objetivos da pesquisa, e a concordância em participar ocorreu mediante assinatura do Termo de Consentimento Livre e Esclarecido. A pesquisa foi aprovada pelo Comitê de Ética em Pesquisa da Universidade Federal de São Paulo, sob o Protocolo n. ${ }^{\circ}$ 0856/08.

\section{RESULTADOS}

Pesquisaram-se os prontuários de 66 pacientes, dos quais oito foram excluídos por não conterem as variáveis de interesse e quatro cujos pacientes não compareceram à consulta. Ao final, foram entrevistados 54 indivíduos, dos quais 36 eram do sexo feminino, 46,3\% não tinham completado o ensino fundamental, 59,3\% responderam ser de cor branca e $57,4 \%$ possuiam renda mensal familiar entre dois e cinco salários mínimos (Tabela 1). A média de idade foi 58 anos e 26 entrevistados eram idosos, dos quais 18 eram do sexo feminino; as médias da pressão arterial $(\mathrm{mmHg})$ sistólica e diastólica foram 136 e 83, respectivamente (Tabela 2 ).

Tabela 1 - Características sociodemográficas de indivíduos hipertensos segundo sexo. São Paulo, jul/set. de 2008

\begin{tabular}{|c|c|c|c|}
\hline Variável & $\begin{array}{c}\text { Masculino } \\
(\mathrm{n}=18)\end{array}$ & $\begin{array}{c}\text { Feminino } \\
(\mathrm{n}=36)\end{array}$ & $\begin{array}{c}\text { Todos } \\
(\mathrm{n}=54)\end{array}$ \\
\hline \multicolumn{4}{|l|}{ Cor/etnia* } \\
\hline Parda & $3(16,7)$ & $13(36,1)$ & $16(29,6)$ \\
\hline Branca & $14(77,7)$ & $18(50,0)$ & $32(59,9)$ \\
\hline Negra & $1(5,6)$ & $4(11,1)$ & $5(9,3)$ \\
\hline Amarela & - & $1(2,8)$ & $1(1,8)$ \\
\hline \multicolumn{4}{|l|}{$\begin{array}{l}\text { Renda familiar mensal } \\
\text { (salário mínimo) }\end{array}$} \\
\hline$<2$ & $6(33,3)$ & $14(38,9)$ & $20(37,0)$ \\
\hline $2-5$ & $10(55,6)$ & $21(58,3)$ & $31(57,4)$ \\
\hline$>5$ & $2(11,1)$ & $1(2,8)$ & $3(5,6)$ \\
\hline \multicolumn{4}{|l|}{ Escolaridade } \\
\hline $\begin{array}{l}\text { Ensino fundamental } \\
\text { incompleto }\end{array}$ & $6(33,3)$ & $19(52,8)$ & $25(46,3)$ \\
\hline $\begin{array}{l}\text { Ensino fundamental } \\
\text { completo ou mais }\end{array}$ & $12(66,7)$ & $17(47,2)$ & $29(53,7)$ \\
\hline
\end{tabular}

* Segundo a Pesquisa Nacional por Amostragem de Domicílio (PNAD 2006).
Apenas 28 pacientes apresentavam PA controlada (PAS $<140 \mathrm{mmHg}$, PAD $<90 \mathrm{mmHg}$ ), para todos estes havia sido prescrita medicação, além de dieta hipossódica para 21 deles. Entre os indivíduos obesos $(\mathrm{n}=20)$ e com sobrepeso ( $n=15), 50,0 \%$ e $66,7 \%$, respectivamente, apresentavam PA controlada.

Do total de entrevistados, 64,8\% tinham algum grau de excesso de peso (Tabela 3). Quando estratificados por idade, $46,4 \%$ dos adultos e $26,9 \%$ dos idosos eram obesos.

Quanto aos hábitos de vida, 51,8\% dos entrevistados referiram fazer atividade física, 94,4\% não fumavam e $83,3 \%$ não consumiam bebida alcoólica.

Comparando-se as orientações prescritas com aquelas que os pacientes citaram ter recebido, houve concordância em 37 casos.

Segundo os prontuários, a orientação para adoção de dieta hipossódica foi feita para 42 pacientes, mas 49 entrevistados citaram ter recebido esta orientação e, destes, 20 referiram ter dificuldade em seguí-la, sobretudo por não gostarem de comida sem sal $(35,0 \%)$ ou porque recebiam sua alimentação com sal preparada por outra pessoa $(15,0 \%)$.

Embora tenha sido prescrita atividade física para 24 pacientes, 36 mencionaram ter recebido esse tipo de orientação e, destes, apenas 16 disseram fazer caminhada, em média quatro vezes por semana. Do total de entrevistados, 12 referiram dificuldades para realizar atividade física, e as principais razões foram dor $(25,0 \%)$, falta de companhia $(16,7 \%)$ e falta de tempo $(16,7 \%)$.

Em relação à terapia medicamentosa, 12 pacientes disseram ter dificuldades para seguir o tratamento e a principal razão foi lembrar o horário para tomar a medicação $(66,7 \%)$. De todos os entrevistados, apenas um paciente não havia recebido orientação para uso de medicamento anti-hipertensivo.

Ao se estratificar por grupo etário, observou-se que todas as cinco citações referentes à falta de tempo, por conta do trabalho, para aderir ao tratamento foram feitas por indivíduos adultos, e das oito citações relativas à dificuldade para lembrar o horário para tomar a medicação, sete foram feitas por idosos. Daqueles 20 pacientes que referiram dificuldade para seguir dieta hipossódica, metade

Tabela 2 - Características antropométricas e valores de pressão arterial, segundo sexo e idade. São Paulo, jul/set. de 2008

\begin{tabular}{|c|c|c|c|c|c|}
\hline Variável & $\begin{array}{l}\text { Todos } \\
(\mathrm{n}=54)\end{array}$ & $\begin{array}{l}\text { Masculino } \\
\quad(\mathrm{n}=18)\end{array}$ & $\begin{array}{l}\text { Feminino } \\
(\mathrm{n}=36)\end{array}$ & $\begin{array}{c}\text { Adulto }(<60 \text { anos } \\
(\mathrm{n}=28)\end{array}$ & $\begin{array}{c}\text { doso }(\geq 60 \text { anos }) \\
(\mathrm{n}=26)\end{array}$ \\
\hline Idade (anos) & $58,0 \pm 12,8$ & $59,4 \pm 12,5$ & $57,3 \pm 13,1$ & $48,0 \pm 7,8$ & $68,7 \pm 7,3$ \\
\hline Peso & $76,0 \pm 17,0$ & $79,6 \pm 14,9$ & $74,2 \pm 17,8$ & $80,0 \pm 16,4$ & $71,8 \pm 16,8$ \\
\hline Altura (m) & $1,60 \pm 0,08$ & $1,66 \pm 0,06 *$ & $1,58 \pm 0,08$ & $1,63 \pm 0,08 * *$ & $1,58 \pm 0,06$ \\
\hline $\operatorname{IMC}\left(\mathrm{kg} / \mathrm{m}^{2}\right)$ & $29,5 \pm 6,0$ & $28,9 \pm 4,7$ & $29,7 \pm 6,6$ & $29,9 \pm 5,0$ & $28,9 \pm 7,0$ \\
\hline PAS (mm Hg) & $136 \pm 16$ & $139 \pm 11$ & $135 \pm 18$ & $134 \pm 11$ & $138 \pm 21$ \\
\hline $\mathrm{PAD}(\mathrm{mm} \mathrm{Hg})$ & $83+11$ & $86+13$ & $92+9$ & $84+9$ & $83+13$ \\
\hline
\end{tabular}

$* \mathrm{p}<0,001$ masculino $v$ s feminino $* * \mathrm{p}<0,05$ adulto $v$ s idoso 
era idosa, bem como daqueles 12 pacientes que relataram dificuldade para realizar atividade física.

A média do tempo de diagnóstico do grupo de pacientes que disse saber o que é hipertensão arterial $(77,8 \%)$, foi nove anos e entre aqueles que não sabiam, foi oito anos.

Observou-se associação entre o conhecimento sobre hipertensão arterial e o seguimento das orientações recebidas. Não se notou associação entre as demais variáveis investigadas.

A resposta "é a pressão alta" à pergunta "o que o (a) senhor (a) sabe sobre hipertensão arterial?" foi dada por $48,1 \%$ dos pacientes.

As principais doenças associadas, referidas pelos pacientes, foram: diabetes $(\mathrm{n}=28)$ e hipercolesterolemia $(\mathrm{n}=13)$. Embora 20 pacientes tenham sido classificados como obesos, apenas seis referiram ter a obesidade como doença associada.

Tabela 3 - Classificação de pacientes hipertensospor estado nutricional, segundo sexo. São Paulo, jul/set. de 2008

\begin{tabular}{lccc}
\hline $\begin{array}{l}\text { Estado } \\
\text { nutricional }\end{array}$ & Masculino (\%) & Feminino (\%) & Total (\%) \\
\hline Baixo peso & $1(5,6)$ & $3(8,3)$ & $4(7,4)$ \\
Eutrofia & $5(27,8)$ & $10(27,8)$ & $15(27,8)$ \\
Sobrepeso & $6(33,3)$ & $9(25,0)$ & $15(27,8)$ \\
Obesidade & $6(33,3)$ & $14(38,9)$ & $20(37,0)$ \\
\hline Total & $18(100)$ & $36(100)$ & $54(100)$ \\
\hline
\end{tabular}

\section{DISCUSSÃO}

O controle da PA entre os indivíduos hipertensos, mesmo que medicados, é algo difícil. No Brasil, alguns pesquisadores $^{(12-14)}$ encontraram entre $20 \%$ e $35 \%$ de hipertensos controlados. No presente estudo, $51,8 \%$ dos pacientes tinham PA controlada. No entanto, os valores da PA considerados neste estudo foram aqueles registrados no dia da entrevista e podem não representar os valores reais, pois $14,8 \%$ dos pacientes referiram esquecer os horários corretos da medicação. Embora não tenha sido perguntado, é possível que os pacientes tenham se lembrado de tomar a medicação no dia anterior à consulta médica, e isto tenha se refletido nos níveis da PA.

Neste estudo, 20 pacientes eram obesos, mas apenas seis citaram ter a obesidade como doença associada à hipertensão. Isto sugere que alguns indivíduos não consideram ou desconhecem que a obesidade é uma doença e um fator de risco para hipertensão e, portanto, deve ser tratada, o que favorece o controle da hipertensão. As modificações no estilo de vida e a prática de hábitos saudáveis, como por exemplo, a adoção de uma alimentação equilibrada e a realização regular de atividade física com o objetivo de controle do peso corporal, entre outros, são fundamentais para prevenção e tratamento da hipertensão ${ }^{(1)}$.

Grande parte das pesquisas aborda a adesão à prescrição medicamentosa, mas as mudanças dos hábitos alimentares e de estilo de vida, isto é, o tratamento não medicamentoso é preconizado a todos os pacientes, independente do risco cardiovascular e do estágio de hipertensão ${ }^{(1)}$. Contudo, segundo os prontuários pesquisados, 22,2\% dos pacientes não receberam orientação para adotar uma dieta hipossódica e apenas $44,4 \%$ foram orientados a realizar atividade física, e 64,8\% dos entrevistados apresentavam variáveis graus de excesso de peso. Outros pesquisadores ${ }^{(15)}$ observaram que $18,4 \%$ dos pacientes entrevistados relataram como dificuldade a falta de instruções quanto à mudança no estilo de vida e que $47 \%$ seguiam apenas o tratamento medicamentoso.

No presente estudo, observou-se que a principal dificuldade no tratamento da hipertensão relatada pelos entrevistados é a adoção de uma dieta hipossódica. Outros pesquisadores ${ }^{(16)}$ observaram que $24 \%$ dos hipertensos entrevistados ainda consumiam regularmente alimentos com alto teor de sódio e resultados mais preocupantes foram encontrados em um outro estudo ${ }^{(12)}$, no qual mais de $50 \%$ dos hipertensos não faziam restrição de sal na alimentação. Analisando os resultados da Pesquisa de Orçamento Familiar realizada, em 2003, os pesquisadores ${ }^{(17)}$ observaram uma disponibilidade de $4,5 \mathrm{~g}$ de sódio para consumo diário por pessoa no Brasil, excedendo o limite máximo diário recomendado de $2 \mathrm{~g}$ para prevenção da hipertensão ${ }^{(18)}$. Considerando esses resultados, conclui-se que a mudança para um padrão alimentar mais adequado para o tratamento da hipertensão é um processo que requer bastante esforço e colaboração de todos. É importante destacar que os alimentos industrializados são importantes fontes de sódio e não apenas o sal de cozinha.

O excesso de peso é um fator de risco para lesões e dor no sistema musculoesquelético, o que pode limitar a prática regular de atividade física ${ }^{(19)}$, conforme se observou pelos relatos dos entrevistados. Nesse sentido, é importante que os pacientes sejam orientados a realizar atividade física que não sobrecarregue o sistema musculoesquelético.

Embora a renda familiar e a condição socioeconômica sejam descritas, como fatores associados negativamente à adesão ao tratamento de doenças crônicas ${ }^{(8)}$, não se observou esta associação no presente estudo. Contudo, dois entrevistados que referiram dificuldade para aderir ao tratamento por falta de recursos financeiros para adquirir a medicação não disponível no Centro de Saúde ou para ter uma alimentação mais adequada estavam no grupo que referiu renda familiar inferior a dois salários mínimos.

Nestes estudos, as medidas antropométricas bem como as da PA utilizadas foram coletadas baseadas nas informações dos prontuários, o que constitui uma das limitações do estudo, pois sabe-se que as técnicas para se obter estas medidas devem ser padronizadas para diminuir o erro de medidas e, consequentemente, a 
classificação do estado nutricional e de hipertensão dos indivíduos.

Algumas limitações deste estudo devem ser citadas, como por exemplo, a falta de confirmação das doenças associadas, que foram autorreferidas, bem como o tratamento proposto para as mesmas. No entanto, sabese que as principais doenças associadas à hipertensão são as que foram referidas pelos pacientes, isto é, diabetes e hipercolesterolemia. Além disso, observou-se alta frequência de paciente hipertenso com excesso de peso, assim como em outros estudos ${ }^{(13,20)}$. A situação é bastante preocupante, pois as dificuldades que os pacientes encontram para tratar a hipertensão aplicam-se às demais doenças, ou seja, mudar os hábitos alimentares e praticar regularmente atividade física.

Embora outros autores ${ }^{(20)}$ tenham encontrado associação entre as variáveis sociodemográficas e os níveis de PA, não se observou esta associação neste estudo, possivelmente, decorrente de um número pequeno de indivíduos entrevistados.

As discordâncias entre as orientações feitas, segundo os prontuários, e aquelas referidas pelos pacientes, indicam claramente que se faz necessário melhorar a comunicação entre os envolvidos no tratamento da hipertensão, e sugerem a importância de investigações futuras sobre a qualidade dos registros nos prontuários, considerando que estes são fontes de informações essenciais sobre a condução e o resultado do tratamento proposto, e que possam gerar comportamento mais próativo por parte dos profissionais que lidam com hipertensão.

É imprescindível identificar as dificuldades com vistas a uma melhor adesão ao tratamento da hipertensão, bem como das doenças crônicas associadas, destacando a obesidade, que não foi percebida como tal pelos pacientes deste estudo, reforçando a necessidade de se informar e discutir as condições observadas pelos membros da equipe de saúde.

É importante destacar que o fator tempo foi citado como um limitante à adesão ao tratamento entre os adultos e, entre os idosos, não lembrarem os horários da medicação. Convém aos profissionais envolvidos no tratamento da hipertensão conhecer estas informações, pois sugerem a prioridade dada ao trabalho pelo adulto, em detrimento de sua condição de saúde, e a dependência que o idoso tem de ser responsável para lhe administrar a medicação.

Embora os pacientes demonstrem ter conhecimento

\section{REFERÊNCIAS}

1. Sociedade Brasileira de Cardiologia, Sociedade Brasileira de Hipertensão, Sociedade Brasileira de Nefrologia. V Diretrizes Brasileiras de Hipertensão Arterial. [texto na internet]. São Paulo: 2006. [citado 2009 fev 26]. Disponível em http:/ / publicacoes.cardiol.br/consenso/2006/VDiretriz-HA.pdf 2. Lopez AD, Mathers CD, Ezzati M, Jamison DT, Murray CJ. da doença, não se observou associação entre o conhecimento ou o seguimento das orientações com o controle da PA, como observado por outros pesquisadores ${ }^{(14)}$, o que mostra que é necessário mais do que o conhecimento para se atingir o controle adequado da PA. A colaboração dos familiares, uma melhor comunicação entre os profissionais e os pacientes e orientações mais fáceis de serem compreendidas, entre outras ações, favorecem a adesão ao tratamento ${ }^{(9)} \mathrm{e}$, possivelmente, o controle da hipertensão.

\section{CONCLUSÃO}

As modificações no estilo de vida, isto é, a adoção de dieta hipossódica e a realização regular de atividade física configuraram-se como a principal dificuldade para a adesão ao tratamento para hipertensão proposto pela equipe de saúde.

Claramente apenas orientações e distribuição gratuita da medicação não foram suficientes para garantir a adesão ao tratamento, considerando as dificuldades relatadas.

A educação em saúde objetiva a conscientização do paciente para a necessidade de modificar o estilo de vida, além de entender e conhecer o tratamento e favorecer um comportamento participativo. Nesse sentido, as orientações devem ser adequadamente registradas e feitas de forma clara e simples, o que pode evitar as discordâncias entre registros e relatos, como as observadas no presente estudo, e a equipe de saúde deve ser ampliada, contando com a participação de educadores físicos e psicólogos, entre outros profissionais, a fim de elaborar alternativas, com os pacientes e os membros da família às dificuldades apontadas.

Além dos pacientes, cuidadores, familiares e membros da equipe de saúde, devem se sentir incluídos pesquisadores, governo e sociedades médicas e afins na discussão sobre o tratamento da hipertensão, as dificuldades enfrentadas e a busca por meios de resolvêlas, com o objetivo de aumentar a adesão ao tratamento e possibilitar um melhor controle da hipertensão.

\section{AGRADECIMENTOS}

As autoras agradecem a colaboração das nutricionistas Fernanda Sanches Carvalho e Priscila Regina Bolelli Broinizi e a colaboração e o apoio da equipe de saúde do Centro de Saúde Dr. Lívio Amato.

Global and regional burden of disease and risk factors, 2001: systematic analysis of population health data. Lancet. 2006;367(9524):1747-57. Comment in: Lancet. 2006;368(9533):365.

3. World Health Organization. The world health report 2002. Reducing risks, promoting healthy life. Geneva: WHO; 2002. 
[cited 2008 May 14]. Available from: http:/ /www.who.int/ whr/2002.

4. Passos VMA, Assis TD, Barreto SM. Hipertensão arterial no Brasil: estimativa de prevalência a partir de estudos de base populacional. Epidemiol Serv Saúde. 2006;15(1):3545.

5. Mochel EG, Andrade CF, Almeida DS, Tobias AF, Cabral R, Cossetti RD. Avaliação do tratamento e controle da hipertensão arterial sistêmica em pacientes da rede pública em São Luís (MA). Rev Baiana Saúde Pública. 2007;31(1):90-101.

6. Castro RAA, Moncau JEC, Marcopito LF. Prevalência de hipertensão arterial sistêmica na cidade de Formiga, MG. Arq Bras Cardiol. 2007;88(3):334-9.

7. Falcão LM, Guedes MVC, Silva LF. Portador de hipertensão arterial: compreensão fundamentada no Sistema Pessoal de Imogene King. Rev Paul Enferm. 2006;25(1):44-50.

8. World Health Organization. Adherence to long-term therapies: evidence for action. [texto na internet] Geneva: WHO; 2003. [cited 2008 May 15]. Available from: http:// www.who.int/chp/knowledge/publications/ adherence_full_report.pdf

9. Coelho EB, Nobre F. Recomendações práticas para se evitar o abandono do tratamento anti-hipertensivo. Rev Bras Hipertens. 2006;13(1):51-4.

10. World Health Organization - WHO. Physical status: the use and interpretation of anthropometry. Geneve: World Health Organization; 1995. (Technical Report Series, 854).

11. Marucci MFN, Barbosa AR. Estado nutricional e capacidade física. In: Lebrão ML. SABE: Saúde, Bem-estar e Envelhecimento; o projeto Sabe no município de São Paulo, uma abordagem inicial. Brasília: Organização Pan-Americana da Saúde; 2003. Cap. 3. p.95-117.

12. Mano GMP, Pierin AMG. Avaliação de pacientes hipertensos acompanhados pelo Programa Saúde da Família em um
Centro de Saúde Escola. Acta Paul Enferm. 2005;18(3):26975.

13. Freitas JB, Tavares A, Kohlmann Júnior O, Zanella MT, Ribeiro AB. Estudo transversal sobre o controle da pressão arterial no serviço de nefrologia da Escola Paulista de Medicina - UNIFESP. Arq Bras Cardiol. 2002;79(2):117-28.

14. Strelec MAAM, Pierin AMG, Mion Júnior D. A influência do conhecimento sobre a doença e a atitude frente à tomada dos remédios no controle da hipertensão arterial. Arq Bras Cardiol. 2003;81(4):343-54.

15. Mascarenhas CHM, Oliveira MML, Souza MS. Adesão ao tratamento em grupo de hipertensos do bairro Joaquim Romão - Jequié/BA. Rev Saúde Com. 2006;2(1):30-8.

16. Girotto E. Adesão ao tratamento anti-hipertensivo e fatores associados na área de abrangência de uma unidade de Saúde da Família, Londrina, PR [dissertação]. Londrina: Universidade Estadual de Londrina; 2008.

17. Sarno F, Claro RM, Levy RB, Bandoni DH, Ferreira SRG, Monteiro CA. Estimativa de consumo de sódio pela população brasileira, 2002-2003. Rev Saúde Pública = J Public Health. 2009;43(2):219-25.

18. World Health Organization. Diet, nutrition and the prevention of chronic diseases. Report of a Joint WHO/ FAO Expert Consultation. Geneva; 2003. (WHO Technical report series, 916). [cited 2008 May 15]. Available from: http:/ /www.who.int/dietphysicalactivity/publications/trs916/ en/

19. Wearing SC, Hennig EM, Byrne NM, Steele JR, Hills AP. Musculoskeletal disorders associated with obesity: a biomechanical perspective. Obes Rev. 2006;7(3):239-50.

20. Pierin AMG, Mion Jr D, Fukushima JT, Pinto AR, Kaminaga MM. O perfil de um grupo de pessoas hipertensas de acordo com conhecimento e gravidade da doença. Rev Esc Enferm USP. 2001;35(1):11-8. 